\title{
Influencia del estilo docente en la motivación y estilo de vida de adolescentes en educación física *
}

\section{Influence of Teaching Style on Motivation and Lifestyle of Adolescents in Physical Education}

Alfonso Valero-Valenzuela Universidad de Murcia, España

ORCID: https://orcid.org/0000-0002-4317-1665

Juan Andrés Merino-Barrero Universidad Internacional de La Rioja, España

ORCID: https://orcid.org/0000-0002-0861-0810

David Manzano-SÁNCHez

Universidad de Murcia, España

ORCID: https://orcid.org/0000-0003-2155-4515

Noelia Belando-Pedreño a

Universidad Europea de Madrid, España

ORCID: https://orcid.org/0000-0002-2067-0816

José DAVid Fernández-Merlos

Universidad de Murcia, España

ORCID: https://orcid.org/0000-0001-6931-7020

Juan Antonio Moreno-Murcia

Universidad Miguel Hernández Elche, España

ORCID: https://orcid.org/0000-0002-6912-4859 Autor de correspondencia. Correo
electrónico: noelia.belando@universidadeuropea.es

Para citar este artículo: Valero-Valenzuela, A., MerinoBarrero, J. A., Manzano-Sánchez, D., BelandoPedreño, N., Fernández-Merlos, J. D., \& MorenoMurcia, J. A. (2020). Influencia del estilo docente en la motivación y estilo de vida de adolescentes en educación física. Universitas Psychologica, 19. 1-11. https://doi.org/10.11144/Javeriana.upsy19.iedm

\section{RESUMEN}

El objetivo del estudio fue identificar los perfiles motivacionales en estudiantes de Educación Secundaria y relacionarlos con el estilo docente y su estilo de vida. Se realizó un estudio descriptivo transversal con 388 estudiantes $(M=13.2 ; D E=1.04)$. Las variables analizadas fueron el estilo interpersonal del docente, la satisfacción de las necesidades psicológicas básicas, la motivación hacia la práctica deportiva y el estilo de vida. Los resultados revelaron la existencia de dos perfiles motivacionales en los estudiantes: "autodeterminado" $(n=261)$ con puntuaciones mayores en motivación intrínseca y regulación identificada y un perfil "no autodeterminado" $(n=127)$ con mayores niveles de desmotivación, regulación introyectada y regulación externa. Como conclusión, se destaca la importancia de la actuación del docente en el aula a través del apoyo de la autonomía, con el fin de contribuir a lograr mayores niveles de motivación y un estilo de vida más activo. 
Palabras clave

motivación positiva; estilo interpersonal; actividad física; salud; necesidades psicológicas básicas.

\begin{abstract}
The objective of the study was to identify the motivational profiles in Secondary Education students and relate them to the teaching style and their lifestyle. A cross-sectional descriptive study was carried out with 388 students (M $=13.2, \mathrm{SD}=1.04)$. The variables analyzed were the interpersonal style of the teacher, the satisfaction of basic psychological needs, the motivation towards sports practice and lifestyle. The results revealed the existence of motivational profiles in students: "self-determined" ( $n$ $=261$ ) with greater obstacles in intrinsic motivation and identified regulation and a "non-self-determined" profile $(n=127)$ with higher levels of demotivation, introjected regulation and external regulation. In conclusion, it highlights the importance of the teacher's performance in the classroom through the support of autonomy, in order to contribute to the highest levels of motivation and active lifestyle.
\end{abstract}

Keywords

positive motivation; interpersonal style; physical activity; health; basic psychological needs.

En los últimos tiempos, las nuevas tecnologías de la información y la comunicación han tenido un gran impacto en nuestra sociedad con un aumento del sedentarismo, una reducción de la actividad física y una alimentación menos saludable (Ruiz \& Castañeda, 2016; Tapia-López, 2019). Como consecuencia, cada vez es mayor el tiempo que los jóvenes estudiantes están conectados a internet, videojuegos, televisión, etc. (Oliva et al., 2012). En este sentido, uno de los problemas en la sociedad actual tiene que ver con el uso del tiempo libre y de ocio de los adolescentes, donde existe un alto predominio de actividades sedentarias como el ocio digital (Crespo, Permas, \& Bóo, 2016). Por esta razón, se hace necesario estudiar la motivación de los adolescentes en las clases de Educación Física (EF) en cuanto a la práctica deportiva y demás contenidos del currículo educativo. Son varias las investigaciones que informan de una menor percepción de la aptitud física, percepción de competencia y de gestión de la salud en la población adolescente de $3 .^{\circ}$ y $4 .^{\circ}$ curso de Enseñanza de Secundaria (Duncan et al., 2011;
Granero-Gallegos, Baena-Extremera, SánchezFuentes, \& Martínez-Molina, 2014; SánchezMiguel, Leo, Amado, Pulido, \& SánchezOliva, 2017). Parece que la motivación está condicionada por diversos factores como la etapa de desarrollo de la persona, el estilo interpersonal del docente y el apoyo social (de profesores, padres, etc.). Por ello, la teoría de la autodeterminación (TAD) se muestra como una macroteoría de la motivación humana relacionada con el desarrollo y funcionamiento de la personalidad en contextos sociales (Ryan \& Deci, 2000). Se centra, por tanto, en analizar el origen de la motivación y cómo esta puede dar lugar a diferentes consecuencias cognitivas, conductuales y afectivas en la vida de las personas (Deci \& Ryan, 2012). Al respecto, diversos autores (Fin, Baretta, MorenoMurcia, \& Nodari, 2017; Moreno-Murcia, Cervelló, Huéscar, Belando, \& Rodríguez, 2013) afirman que el docente, en las clases de EF, es uno de los máximos responsables para generar experiencias satisfactorias en el aula, siendo su intervención determinante para la motivación del alumnado. En esta línea, Vallerand (2007) muestra una secuencia en la cual el impacto de los factores sociales (como el estilo interpersonal del profesor) está mediado por las necesidades psicológicas básicas (autonomía percibida, competencia percibida y relación con los demás), que median la motivación más positiva hacia comportamientos como el mantenimiento de estilo de vida activo y saludable. Siguiendo a Ulstad, Halvari, Søreb $\varnothing$ y Deci (2018) y Reeve et al. (2014), los efectos experimentales de una intervención basada en la teoría de la autodeterminación, con estrategias de aprendizaje como la regulación del esfuerzo, implicación de los estudiantes en la toma de decisiones, así como la importancia del rol o estilo docente, es un aspecto fundamental para el desarrollo de un adecuado proceso de enseñanza-aprendizaje en el aula y fuera de ella. El estilo de enseñanza que emplea el docente, implica un continuo que iría desde el perfil del docente "controlador" (tomando este todas las decisiones sobre las tareas de clase, evitando así la participación activa del 
alumnado) hasta uno que genera un gran "apoyo a la autonomía" (facilitando que cada alumno aprenda a su propio ritmo y se sienta implicado en la toma de decisiones sobre las tareas y la organización del grupo de clase). El apoyo a la autonomía de los "referentes de autoridad", como es considerada la figura del docente en el aula, se relaciona con una mayor motivación autodeterminada y la satisfacción de las necesidades psicológicas básicas (Frielink, Schuengel, \& Petri, 2018).

Para conocer el grado de motivación de los jóvenes estudiantes hacia la práctica de actividad físico-deportiva, es preciso analizar los perfiles motivacionales que presentan. Al respecto, Haerens et al. (2018) informan sobre la relación significativa entre el grupo de clase con mayor apoyo a la autonomía, la motivación intrínseca y la satisfacción de las necesidades psicológicas básicas. Por su parte, Kujong (2017) establece que existe una clara relación entre los niveles de motivación más interna y la satisfacción personal, en contraste con los grupos con mayor motivación extrínseca o desmotivación, quienes además expresaron una mayor búsqueda de satisfacción externa. Ng, Liu y Wang (2016) encontraron diversos perfiles motivacionales en estudiantes de secundaria, relacionando los perfiles con sentimientos de autoeficacia, estrategias de aprendizaje y orientación a la tarea, con mayor satisfacción de las necesidades de autonomía, competencia y relación social, mayor motivación intrínseca, calificaciones y percepción de apoyo a la autonomía por parte del profesor. Finalmente, atendiendo al estilo de vida, autores como Friederichs, Oenema, Bolman y Lechner (2015) y Yli-Piipari, Watt, Jaakkola, Liukkonen y Nurmi (2009) afirman que un perfil más autodeterminado se relaciona con un estilo de vida más activo y saludable.

Según lo expuesto, el presente estudio tuvo como principal objetivo identificar los diferentes perfiles motivacionales en los estudiantes y relacionarlos con el estilo interpersonal del docente de EF, así como con la satisfacción de las necesidades psicológicas básicas y el estilo de vida. Se hipotetiza que los estudiantes con un perfil más autodeterminado de motivación, percibirán un estilo interpersonal con mayor apoyo a la autonomía y menor estilo controlador por parte del profesor, y a su vez presentarán una mayor satisfacción de sus necesidades psicológicas básicas y un mayor comportamiento hacia un estilo de vida activo.

\section{Método}

\section{Participantes}

Participaron un total de 388 estudiantes de EF, 217 chicos y 171 chicas, con edades comprendidas entre los 12 y los 17 años $(M=$ 13.2; $D E=1.04)$, pertenecientes a los niveles desde 1. a 4. curso de Educación Secundaria Obligatoria de diferentes Centros Educativos públicos de la región de Murcia.

\section{Medidas}

\section{Apoyo a la autonomía}

Se utilizó la Escala de Apoyo a la Autonomía de Moreno-Murcia, Huéscar, Andrés-Fabra y Sánchez-Latorre (2020). El cuestionario está compuesto por once ítems que los participantes han de responder acerca del estilo del profesor o el entrenador en las clases (e. g., "Con sus explicaciones, nos ayuda a comprender para qué sirven las actividades que realizamos"). La sentencia previa utilizada fue: "En mis clases de educación física, mi profesor/a...”. Consta de una escala Likert de cinco opciones de respuesta, desde (1) Seguro que no hasta (5) Seguro que sí. La escala obtuvo un valor $\alpha=0.8$.

\section{Estilo controlador}

Se utilizó la Escala de Estilo Controlador de Moreno-Murcia et al. (2020). El cuestionario está compuesto por nueve ítems que los participantes han de responder acerca del estilo del profesor o el entrenador en las clases (e. g., "Habla continuamente y no permite que 
realicemos aportaciones en clase"). La sentencia previa utilizada fue: "En mis clases de educación física, mi profesor/a...”. Consta de una escala Likert de cinco opciones de respuesta, desde (1) Seguro que no hasta (5) Seguro que sí. La escala obtuvo un valor $\alpha=0.8$.

\section{Necesidades psicológicas básicas}

Se utilizó la Psychological Need Satisfaction in Exercise Scale (PNSE) de Wilson, Rogers, Rodgers y Wild (2006), validada al contexto español por Moreno-Murcia, Huéscar y Cervelló (2012). La PNSE utiliza 18 ítems, seis para evaluar cada una de las necesidades: competencia (e. g., "Tengo confianza para hacer los ejercicios más desafiantes”), autonomía (e. g., "Creo que puedo tomar decisiones en mis clases de Educación Física”) y relación con los demás (e. g., "Me siento unido a mis compañeros de clase porque ellos me aceptan como soy). La sentencia previa fue "En mis clases de Educación Física..." y las respuestas fueron recogidas en una escala tipo Likert, cuyo rango de puntuación oscila entre 1 (Falso) y 6 (Verdadero). La consistencia interna reveló un valor de $\alpha=0.88, \alpha=0.72$ y $\alpha$ $=0.76$, respectivamente. La escala total obtuvo un valor $\alpha=0.81$.

\section{Motivación}

Se utilizó el cuestionario denominado Cuestionario de Regulación de Conducta en el Deporte (BRSQ) de Lonsdale, Hodge y Rose (2008), validado al español por MorenoMurcia, Marzo, Martínez y Conte (2011). Consta de 36 ítems agrupados en nueve factores de cuatro ítems cada uno que miden la motivación intrínseca general (e. g., "porque lo disfruto"), la motivación intrínseca sobre el conocimiento (e. g., "por el placer que me da el conocer más acerca de este deporte"), la motivación intrínseca de estimulación (e. g., "por el entusiasmo que siento cuando estoy implicado en la actividad"), la motivación intrínseca de consecución (e. g., "porque disfruto cuando intento alcanzar metas a largo plazo"), la regulación integrada (e. g., "porque es parte de lo que soy"), la regulación identificada (e. g., "los beneficios del deporte son importantes para mî”), la regulación introyectada (e. g., "porque me sentiría avergonzado si lo abandono"), la regulación externa (e. g., "porque si no lo hago otros no estarían contentos conmigo") y la desmotivación (e. g., "sin embargo, no sé por qué lo hago). La frase introductoria utilizada fue: "Participo en este deporte...”. Se utilizó una escala tipo Likert de siete puntos que va desde 1 (Muy falso) a 7 (Muy verdadero). La fiabilidad de las variables fue de $\alpha=0.9$ para la motivación general; $\alpha=0.87$ para la motivación intrínseca de conocimiento; $\alpha=0.88$ para la motivación intrínseca de estimulación; $\alpha=0.88$ para la motivación intrínseca de consecución; $\alpha=$ 0.90 para la regulación integrada; $\alpha=0.85$ para la regulación identificada; $\alpha=0.83$ para la regulación introyectada; $\alpha=0.83$ para la regulación externa; 0.81 para la desmotivación. La escala total presentó un valor de $\alpha=$ 0.91. Se calculó el Índice de Autodeterminación (IAD): [ $(2 \mathrm{x}$ (MI hacia el conocimiento + MI hacia la ejecución + MI hacia la estimulación) /3) + Regulación Identificada) ] $[($ Regulación Externa + Introyección) / 2) + (2 x desmotivación)] (Vallerand, 1997). La escala total obtuvo un valor $\alpha=0.79$.

\section{Estilo de vida}

Para medir los hábitos y estilo de vida saludable se utilizó el Test Corto Krece Plus (Serra, Aranceta, \& Rodríguez-Santos, 2003). Está compuesto de dos preguntas: icuántas horas ves la televisión o juegas a videojuegos u ordenador diariamente de promedio? y icuántas horas dedicas a actividades deportivas extraescolares semanalmente? Los participantes deben contestar en una escala Likert de seis puntos que va desde el valor 0 hasta más de 4 horas. La escala total obtuvo un valor $\alpha=0.69$. Este cuestionario permite calificar el estilo de vida del participante en "malo" $(0$ a 3 puntos), "regular" (4 a 6 puntos) o "bueno" (7 puntos) (Edo et al., 2010). 


\section{Diseño y procedimiento}

Se utilizó una metodología descriptiva con diseño transversal (Montero \& León, 2007). Se solicitó el consentimiento informado de los alumnos y sus padres tras la obtención del informe favorable del Comité de Ética de la Universidad de Murcia (1403/2016). Los cuestionarios se administraron en presencia del investigador principal y del docente de EF, en un ambiente calmado, durante 20 minutos. Se garantizó el anonimato y la no incidencia de las respuestas en las notas, tratando de reducir las respuestas por deseabilidad social.

\section{Análisis de datos}

Se realizó un análisis descriptivo (medias, desviaciones estándar, asimetría y curtosis), análisis de correlación y se halló la consistencia interna mediante el coeficiente de alfa de Cronbach de todas las variables contempladas en el estudio.

Se identificaron los diferentes perfiles motivacionales existentes entre los estudiantes. Para ello, se realizaron dos análisis de conglomerados (Hair, Anderson, Tatham, \& Black, 1999), dividiendo la muestra aleatoriamente en dos grupos con el mismo número de participantes. En la submuestra 1 se realizó un análisis jerárquico de clúster con método Ward, utilizando las variables explicadas en el BRSQ relativas a los diferentes tipos de motivación. Posteriormente, se trató de confirmar la solución de perfiles hallada, utilizando un análisis de conglomerados de $\mathrm{K}$ medias en la submuestra 2, verificándose la solución encontrada en la muestra total a través del método K-medias. Se examinaron las características de cada perfil mediante un análisis de varianza multivariado (MANOVA) con la muestra total. La magnitud de las diferencias entre los grupos estudiados se obtuvo mediante el tamaño del efecto. Cohen (1988) muestra el tamaño del efecto como pequeño $\left(\eta^{2}=0.2\right)$, medio $\left(\eta^{2}=0.5\right)$ o grande $\left(\eta^{2}=0.8\right)$. Todos los análisis se ejecutaron con el paquete estadístico SPSS 19.0.

\section{Resultados}

\section{Análisis descriptivo y de correlación}

Se presentan los estadísticos descriptivos, los valores de alfa de Cronbach de las diferentes subescalas y las correlaciones bivariadas para todas las variables objeto de estudio (Tabla 1). Los datos indican una mayor puntuación en el apoyo a la autonomía con respecto al estilo controlador ( $M=3.9$ y 2.29 , respectivamente). La competencia percibida obtuvo puntuaciones altas $(M=4.53)$, seguida de la relación $(M=$ 4.31) y la autonomía $(M=3.94)$. En relación con la motivación, reflejó altos niveles de motivación intrínseca comparativamente con los demás tipos de regulaciones motivacionales. El valor de IAD fue positivo $(M=7.04)$. Por último, el estilo de vida de los participantes reveló un valor medio de 6.04. La gran mayoría de los coeficientes de fiabilidad muestran valores por encima de 0.7 , criterio considerado como aceptable para las escalas de dominio psicológico (Nunnally, 1978) y solo un valor se establece en el rango entre 0.6 y 0.7 , considerado aceptable por autores como Sturmey, Newton, Cowley, Bouras y Holt (2005). El análisis de correlaciones bivariadas arrojó que todas las variables tuvieron una relación significativa entre sí en $p<0.01$.

\section{Tabla 1}

Estadísticos descriptivos y correlaciones bivariadas de la muestra

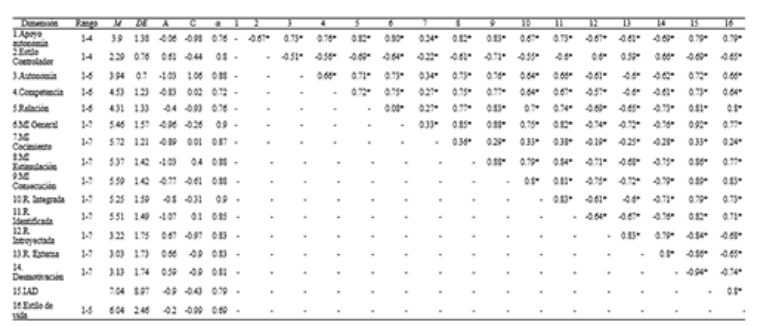

Nota. ${ }^{*} p<0.01 ; M=$ Media DE = Desviación típica; $\mathrm{A}=$ Asimetría; $\mathrm{C}=$ Curtosis; $\alpha$ $=$ Coeficiente alfa de Cronbach; $\mathrm{MI}=$ Motivación Intrínseca; $\mathrm{R}$ = Regulación; IAD = Índice de Autodeterminación. 


\section{Análisis chúster}

El análisis de clúster se llevó a cabo con el objeto de estudiar los perfiles motivacionales, siguiendo las fases propuestas por Hair et al. (1999). Se estandarizaron los valores de las variables utilizando las puntuaciones $Z$, no encontrándose ninguna puntuación por encima de tres, lo que implicó la inexistencia de outliers en la totalidad de la muestra. En el siguiente paso, la distribución univariada de todas las variables agrupadas fue examinada para su normalidad. A continuación, se realizó el agrupamiento de los alumnos en clúster. El dendograma sugirió dos grupos fundamentado en el incremento de los coeficientes de aglomeración al pasar de dos grupos. Se concluyó que existían dos grupos distintos de perfiles motivacionales en la muestra total, un perfil "autodeterminado" (clúster 1) formado por 261 alumnos $(67.3 \%)$ con mayores puntuaciones $Z$ y medias en motivación general, de conocimiento, estimulación, consecución, integrada e identificada y un perfil "no autodeterminado" (clúster 2) formado por 127 alumnos $(32.7 \%)$ con puntuaciones $Z$ y medias más altas en regulación introyectada, externa y desmotivación (Figura 1). En la Tabla 2, se presentan los estadísticos descriptivos, así como los ANOVA para los dos grupos de perfiles motivacionales hallados.

\section{Figura 1}

Análisis de conglomerados jerárquicos con Método Ward en los alumnos estudiados

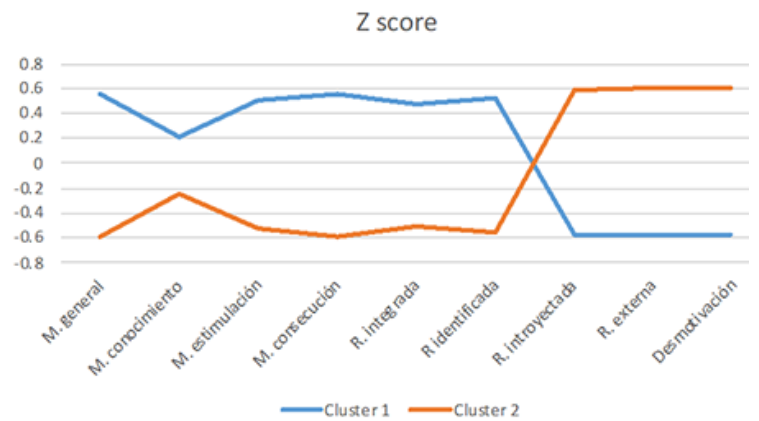

Tabla 2

Medias, desviaciones estándar, puntuaciones $Z$ en cada cluster

\begin{tabular}{lrrrrrrr}
\hline \multirow{2}{*}{ Variables } & \multicolumn{1}{c}{ Clúster $1(n=261)$} & \multicolumn{4}{c}{ Clúster 2} & \multirow{2}{*}{$F$} \\
\cline { 2 - 7 } & \multicolumn{1}{c}{$M$} & $D E$ & \multicolumn{1}{c}{$\mathrm{C}$} & \multicolumn{1}{c}{$\mathrm{DE}$} & \multicolumn{1}{c}{$Z$} & \\
\hline MI conocimiento & 6 & 1.06 & 0.22 & 5.17 & 1.33 & -0.46 & $44.03^{*}$ \\
MI estimulación & 6.1 & 0.77 & 0.51 & 3.9 & 1.31 & -1.04 & $433.7^{*}$ \\
MI consecución & 6.39 & 0.74 & 0.56 & 3.96 & 1.05 & -1.15 & $689.87^{*}$ \\
R integrada & 6.01 & 0.97 & 0.48 & 3.68 & 1.48 & -0.98 & $346.38^{*}$ \\
R identificada & 6.3 & 0.71 & 0.53 & 3.9 & 1.4 & -1.07 & $501.01^{*}$ \\
R introyectada & 2.21 & 0.94 & -0.56 & 5.26 & 1.19 & -1.16 & $748.59^{*}$ \\
R externa & 2.04 & 0.86 & -0.58 & 5.1 & 1.18 & 1.19 & $839.93^{*}$ \\
Desmotivación & 2.14 & 0.93 & -0.57 & 5.19 & 1.11 & 1.18 & $806.89^{*}$ \\
\hline
\end{tabular}

Nota. $M=$ Media; $D E=$ Desviación típica; ${ }^{*} p<0.01 ; \mathrm{MI}=$ Motivación intrínseca; $\mathrm{R}=$ Regulación

\section{Análisis diferencial}

Para analizar las características de cada perfil motivacional de acuerdo a la percepción del alumnado sobre el estilo docente, las necesidades psicológicas básicas y estilo de vida de los alumnos, se realizó un análisis multivariante de la varianza (MANOVA) con los clústeres como variables independientes y el resto de variables como dependientes. Se comprobó la homogeneidad de covarianza, usando la prueba de Box y el estadístico Traza de Pillai para el análisis de los efectos multivariados en caso de que la idea de matriz sea no resuelta $(p<0.05)$ (Tabachnick \& Fidell, 1996). El análisis multivalente (Box, $p<$ 0.05) reveló un efecto significativo a nivel multivariado (Traza de Pillai $=0.58, F_{(381)}=$ $90.82, p<0.01)$. El análisis univariado reflejó puntuaciones significativamente superiores para el grupo "autodeterminado" en la totalidad de las variables analizadas (Tabla 3). 


\section{Tabla 3}

Análisis del estilo interpersonal docente, satisfacción de las necesidades psicológicas básicas y el estilo de vida según el perfil motivacional.

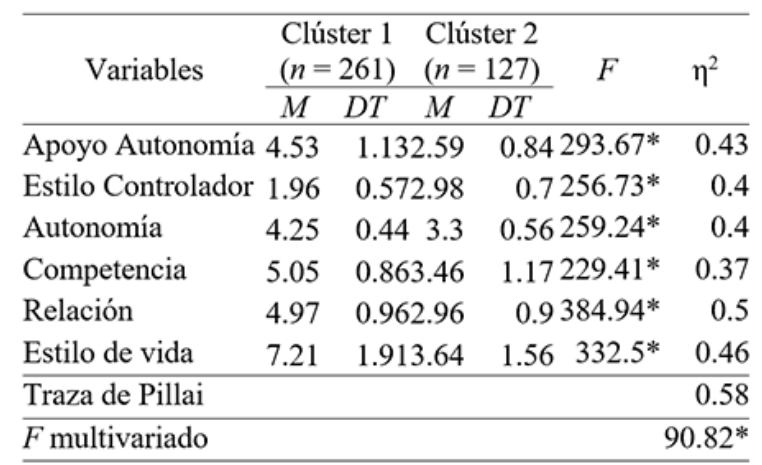

Nota. ${ }^{*} p<0.01 ; M=$ Media; $D E=$ Desviación típica; $\eta^{2}=$ tamaño del efecto de Cohen; Cluster 1 = Autodeterminado;

Cluster 2 = No autodeterminado.

\section{Discusión}

El presente estudio tuvo como primer objetivo identificar los diferentes perfiles motivacionales de jóvenes estudiantes, en relación con la percepción que poseen sobre el estilo interpersonal del docente. La hipótesis del trabajo de investigación ha sido confirmada en cuanto que el grupo de alumnos con un "perfil motivacional más autodeterminado" valora el estilo interpersonal de apoyo a la autonomía del docente.

Los resultados obtenidos revelaron la existencia de dos perfiles motivacionales en los estudiantes: un perfil "autodeterminado", con puntuaciones mayores en motivación intrínseca y regulación identificada que en regulación introyectada, externa y desmotivación. Un perfil "no autodeterminado" con mayores niveles de desmotivación, regulación introyectada y regulación externa hacia la EF. Los hallazgos obtenidos confirman que "el perfil motivacional autodeterminado" se relaciona positivamente con el apoyo a la autonomía por parte del docente, con la satisfacción de las necesidades psicológicas básicas y el estilo de vida. En esta misma línea de investigación, MorenoMurcia, Hellín, González-Cutre y Martínez
(2011) confirmaron que aquellos estudiantes con mayores niveles de motivación presentan mejor predisposición hacia la práctica deportiva y persistencia en la misma. Asimismo, un reciente estudio de Ulstad et al. (2018) demuestra que un programa de intervención diseñado para ayudar a los profesores a ser más autónomos y apoyar al alumnado en la enseñanza de "estrategias de aprendizaje" (en comparación con la enseñanza tradicional) aumentó la percepción de apoyo a la autonomía de los estudiantes respecto a los profesores, la competencia percibida, la motivación autónoma, el esfuerzo, la participación proactiva e incluso se observaron calificaciones más altas en la asignatura de Educación Física, a lo largo de un año escolar.

Se pone de manifiesto como el perfil de los estudiantes con mayores niveles de percepción de apoyo a la autonomía, favorece la motivación más autodeterminada y el interés por participar de un estilo de vida más saludable (Friederichs et al., 2015). Al respecto, Fin et al. (2017) encontraron que estudiantes adolescentes con un alto perfil motivacional intrínseco, se percibían más competentes, más autónomos en la práctica y se relacionaban mejor entre iguales, cuando el docente facilitaba la posibilidad de elegir sobre alguna parte de las tareas en las clases de Educación Física. Por su parte, Chang, Chen, Tu y Chi (2016), tras la aplicación de un diseño de estudio cuasi-experimental que evaluaba el efecto a apoyo a la autonomía sobre la motivación de jóvenes estudiantes de EF, mostraron que la motivación intrínseca fue mayor en el grupo experimental (en el que se apoyaba a la autonomía del alumnado) que en el grupo de control.

En cuanto a la actividad física realizada, los datos de esta investigación coinciden con el estudio llevado a cabo por Yli-Piipari et al. (2009), quienes afirman que los practicantes con un perfil más alto de motivación son más activos físicamente que aquellos estudiantes con un perfil "bajo de motivación", llegando a superar las tres horas de actividad física semanales fuera del aula. Recientemente, Trigueros, Navarro, AguilarParra y León-Estrada (2019) contrastaban en estudiantes españoles de entre 14 y 19 años 
$(N=615)$ que el análisis de las emociones que estos experimentan durante las clases de $\mathrm{EF}$, produce mejores resultados académicos y mantiene hábitos de vida más saludables y activos.

Por otro lado, se analizaron las distintas variables y los perfiles motivacionales, observando cómo los resultados se relacionan con los aportados por Moreno-Murcia y SánchezLatorre (2016), demostrando que los estudiantes que percibieron mayor apoyo a la autonomía obtenían incrementos significativos en sus necesidades psicológicas básicas, motivación e intención de práctica y actividad física habitual.

Como principales limitaciones del estudio, el tipo de muestra empleada fue elegida por accesibilidad y no de forma aleatorizada, comprometiendo así la validez externa. Otro aspecto que se debe considerar es el número de participantes y el diseño del estudio que fue observacional y descriptivo, de manera que futuros estudios han de considerar la posibilidad de realizar mediciones similares en diseños longitudinales, teniendo en cuenta la forma de seleccionar la muestra. Además, son necesarios estudios de carácter experimental para comprobar las relaciones causales entre las variables analizadas. Asimismo, el registro de datos implica una serie de limitaciones dado que la eficacia depende en cierta medida de la capacidad lectora, la sinceridad o la búsqueda de los encuestados de "intentar dar respuestas correctas".

\section{Conclusiones}

Para concluir, tal y como se hipotetizaba, se comprobó que los alumnos con un perfil más autodeterminado presentaban un mayor nivel de percepción de apoyo a la autonomía, mayores niveles de motivación intrínseca y un estilo de vida más saludable, existiendo una relación positiva entre el apoyo a la autonomía percibido por el estudiante, su nivel de motivación autodeterminada hacia el deporte y un estilo de vida saludable.

\section{Referencias}

Chang, Y. K., Chen, S., Tu, K. W., \& Chi, L. K. (2016). Effect of autonomy support on self-determined motivation in elementary physical education. Journal of Sports Science and Medicine, 15(3), 460-466. Recuperado de https://scholar.lib.ntnu.edu.tw/en/publi cations/effect-of-autonomy-support-on-self -determined-motivation-in-eleme

Cohen, J. (1988). Statistical power analysis for the behavioral sciences (2.a ed.). Hillsdate, NJ: LEA.

Crespo, L. V., Pernas, R. G., \& Bóo, Y. T. (2016). Ocio y usos del tiempo libre en adolescentes de 12 a 16 años en España. Educação e Pesquisa, 42 (4), 987-999. https://doi.org/10 $.1590 / \mathrm{s} 1517-9702201612152404$

Deci, E. L., \& Ryan, R. M. (2012). Selfdetermination theory. En P. A. M. Van Lange, A. W. Kruglanski \& E. T. Higgins (Eds.), Handbook of theories of social psychology (Vol. 1, pp. 416-437). Thousand Oaks, CA: Sage.

Duncan, D. T., Wolin, K. Y., Scharoun-Lee, M., Ding, E. L., Warner, E. T., \& Bennet, G. G. (2011). Does perception equal reality? Weight misperception in relation to weight-related attitudes and behaviors among overweight and obese US adults. International Journal of Behavioral Nutrition and Physical Activity, 22, 8-20. https://doi.o $\mathrm{rg} / 10.1186 / 1479-5868-8-20$

Edo, Á., Montaner, I., Bosch, A., Casademont, M. R., Fábrega, M. T., Fernández, Á., ... Torres, O. (2010). Estilos de vida, hábitos dietéticos y prevalencia del sobrepeso y la obesidad en una población infantil. Pediatría Atención Primaria, 12(45), 53-65. Recuperado de http://scielo.isciii.es/scielo.php?script $=$ s ci_arttext\&pid $=$ S1139-763220100001000 $0 \overline{6}$

Friederichs, S. A. H., Oenema, A., Bolman, C., \& Lechner, L. (2015). Long-term effects of self-determination theory and motivational interviewing in a web-based physical activity intervention: Randomized 
controlled trial. International Journal of Behavioral Nutrition and Physical Activity, 12(101), 1-13. https://doi.org/10.1186/s129 66-015-0262-9

Fin, G., Baretta, E., Moreno-Murcia, J. A., \& Nodari Júnior, R. J. (2017). Autonomy support, motivation, satisfaction and physical activity level in physical education class. Universitas Psychologica, 16(4), 1-12. https://doi.org/10.11144/Javeriana.up sy16-4.asms

Frielink, N., Schuengel, C., \& Petri, J. C. M. (2018). Autonomy support, need satisfaction, and motivation for support among adults with intellectual disability: Testing a Self-Determination Theory Model. American Journal on Intellectual and Developmental Disabilities, 123(1), 33-49. ht tps://doi.org/10.1352/1944-7558-123.1.33

Granero-Gallegos, A., Baena-Extremera, A., Sánchez-Fuentes, J. A., \& Martínez-Molina, M. (2014). Perfiles motivacionales de apoyo a la autonomía, autodeterminación, satisfacción, importancia de la educación física e intención de práctica física en el tiempo libre. Cuadernos de Psicología del Deporte, 14(2), 59-70. https://doi.org/10.43 21/S1578-84232014000200007

Haerens, L., Vansteenkiste, M., De Meester, A., Delrue, J., Tallir, I., Vande Broek, G., ... Aelterman, N. (2018). Different combinations of perceived autonomy support and control: Identifying the most optimal motivating style. Physical Education and Sport Pedagogy, 23(1), 16-36. https://do i.org/10.1080/17408989.2017.1346070

Hair, J., Anderson, R., Tatham, R., \& Black, W. (1999). Análisis multivariante (Vol. 491). Madrid: Prentice Hall.

Kujong, Y. (2017). Clustering and case analysis applying the young children motivation types assessment tool. Journal of Children's Literature and Education, 18(1), 241-270. h ttps://doi.org/10.22154/JCLE.18.1.11

Lonsdale, C., Hodge, K., \& Rose, E. A. (2008). The Behavioral Regulation in Sport Questionnaire (BRSQ): Instrument development and initial validity evidence. Journal of Sport and Exercise Psychology, 30(3), 323-355. https://doi.org/10.1123/jse p.30.3.323

Montero, I., \& León, O. (2007). A guide for naming research studies in psychology. International Journal of Clinical and Health Psychology, 7(3), 847-862. Recuperado de http://www.redalyc.org/articulo.oa?id= 33770318

Moreno-Murcia, J. A., Cervelló, E., Huéscar, E., Belando, N., \& Rodríguez, J. (2013). Motivational profiles in physical education and their relation to the Theory of Planned Behavior. Journal of Sports Science Medicine, 12(3), 551-558. Recuperado de https://www.ncbi.nlm.nih.gov/pmc/artic les/PMC3772601/

Moreno-Murcia, J. A., Hellín, P., GonzálezCutre, D., \& Martínez-Galindo, C. (2011). Influence of perceived sport competence and body attractiveness on physical activity and other healthy lifestyle habits in adolescents. Spanish Journal of Psychology, 14, 282-292. https://doi.org/10.5209/rev_S JOP.2011.v14.n1.25

Moreno-Murcia, J., Huéscar, E., Andrés-Fabra,J., \& Sánchez-Latorre, F. (2020). Adaptación y validación de los cuestionarios de apoyo a la autonomía y estilo controlador a la educación física: relación con el feedback. Revista Ciencias de la Actividad Física UCM, 21 (1), 1-16. https://doi.org/10.29035/rcaf.2 1.1 .3

Moreno-Murcia, J., Huéscar, E., \& Cervelló, E. (2012). Prediction of Adolescents doing Physical Activity after Completing Secondary Education. The Spanish Journal of Psychology, 15(1), 90-100. https://doi.org /10.5209/rev_SJOP.2012.v15.n1.37288

Moreno-Murcia, J. A., \& Sánchez-Latorre, F. (2016). The effects of autonomy support in physical education classes. RICYDE. Revista Internacional de Ciencias del Deporte, 12 (43), 79-89. https://doi.org/10.5232/ricyde2016. 04305

Moreno-Murcia, J. A., Marzo, J. C., MartínezGalindo, C., \& Conte, L. (2011). 
Validación de la Escala de Satisfacción de las Necesidades Psicológicas Básicas y del Cuestionario de la Regulación Conductual en el Deporte al contexto español. RICYDE. Revista Internacional de Ciencias del Deporte, 26(7), 355-369. https: //doi.org/10.5232/ricyde2011.02602

Nunnally, J. (1978). Psychometric theory. Nueva York: McGraw-Hill.

Ng, B. L., Liu, W. C., \& Wang, J. C. (2016). Student motivation and learning in mathematics and science: A cluster analysis. International Journal of Science and Mathematics Education, 14(7), 1359-1376. h ttps://doi.org/10.1007/s10763-015-9654-1

Oliva, A., Hidalgo, M. V., Moreno, C., Jiménez, L., Jiménez, A., Antolín, L., \& Ramos, P. (2012). Uso y riesgo de adicciones a las nuevas tecnologías entre adolescentes y jóvenes andaluces. Sevilla: Aguaclara.

Reeve, J., Vansteenkiste, M., Assor, A., Ahmad, I., Cheon, S. H., Jang, H., ... Wang, C. J. (2014). The beliefs that underlie autonomy-supportive and controlling teaching: A multinational investigation. Motivation and Emotion, 38(1), 93-110. https://doi.org/10.1007/s110 31-013-9367-0

Ruiz, R. D., \& Castañeda, M. A. (2016). Relación entre uso de las nuevas tecnologías y sobrepeso infantil, como problema de salud pública. RqR Enfermería Comunitaria, 4(1), 46-51. Recuperado de https://dialnet.unirio ja.es/servlet/articulo?codigo $=5353331$

Ryan, R., \& Deci, E. L. (2000). La teoría de la autodeterminación y la facilitación de la motivación intrínseca, el desarrollo social y el bienestar. American Psychologist, 55(1), 68-78. https://doi.org/10.103711000 3-066X.55.1.68

Sánchez-Miguel, P. A., Leo, F. M., Amado, D., Pulido, J. J., \& Sánchez-Oliva, D. (2017). Relationships between physical activity levels, self-identity, body dissatisfaction and motivation among Spanish high school students. Journal of Human Kinetics, 59, 29-38. https://doi.org/10.1515/hukin-20170145
Serra, M., Aranceta, J., \& Rodríguez-Santos, F. (Eds.). (2003). Crecimiento y desarrollo: Estudio EnKid Krece Plus. Barcelona: Masson.

Sturmey, P., Newton, J. T., Cowley, A., Bouras, N., \& Holt, G. (2005). The PAS-ADD checklist: Independent replication of its psychometric properties in a community sample. British Journal of Psychiatry, 186, 319-323. https://doi.org/10.1192/bjp.186.4. 319

Tabachnick, B., \& Fidell, L. (1996). Using multivariate statistics. Nueva York: Harper Collins.

Tapia López, A. (2019). Diferencias en los niveles de actividad física, grado de adherencia a la dieta mediterránea y autoconcepto físico en adolescentes en función del sexo (Gender differences in physical activity levels, degree of adherence to the Mediterranean diet, and physi). Retos, 36(36), 185-192. Recuperado de https://recyt.fecyt.es/index. php/retos/article/view/67130

Trigueros, R., Navarro, N., Aguilar-Parra, J., \& León-Estrada, I. (2019). Influencia del docente de Educación Física sobre la confianza, diversión, la motivación y la intención de ser físicamente activo en la adolescencia. Cuadernos de Psicología del Deporte, 19. 222-232. https://doi.org/10.60 18/cpd.347631

Vallerand, R. J. (1997). Toward a hierarchical model of intrinsic and extrinsic motivation. Advances in Experimental and Social Psychology, 29, 271-360. https://doi.org/10. 1016/S0065-2601(08)60019-2

Vallerand, R. J. (2007). Intrinsic and extrinsic motivation in sport and physical activity. En G. Tenenbaum \& R. C. Eklund (Eds.), Handbook of Sport Psychology (3.a ed., pp. 59-83). Nueva York: Wiley. https://doi.org/ 10.1002/9781118270011.ch3

Ulstad, S. O., Halvari, H., Sørebø, Ø., \& Deci, E. L. (2018). Motivational predictors of learning strategies, participation, exertion, and performance in physical education: A randomized controlled trial. Motivation and 
Emotion, 42(4), 497-512. https://doi.org/10 $.1007 / \mathrm{s} 11031-018-9694-2$

Wilson, P., Rogers, W., Rodgers, W., \& Wild, T. C. (2006). The Psychological Need Satisfaction in Exercise scale. Journal of Sport and Exercise Psychology, 28, 231-251. h ttps://doi.org/10.1123/jsep.28.3.231

Yli-Piipari, S., Watt, A., Jaakkola, T., Liukkonen, J., \& Nurmi, J. E. (2009). Relationships between physical education students' motivational profiles, enjoyment, state anxiety, and self-reported physical activity. Journal of Sports Science and Medicine, 8, 327-336. Recuperado de https://www.ncbi. nlm.nih.gov/pmc/articles/PMC5680684/

\section{Notas}

* Artículo de investigación. 